06

\title{
Влияние типа проводимости и уровня легирования кристаллов кремния на размеры каналов пор, формирующихся в них при анодном травлении в растворах плавиковой кислоты
}

\author{
(C) Г.Г. Зегря, ${ }^{1}$ В.П. Улин, ${ }^{1}$ А.Г. Зегря, ${ }^{1}$ Н.В. Улин, ${ }^{1}$ В.М. Фрейман, ${ }^{1}$ Ю.М. Михайлов ${ }^{2}$ \\ ${ }^{1}$ Физико-технический институт им. А.Ф. Иофрер РАН, \\ 194021 Санкт-Петербург, Россия \\ ${ }^{2}$ Институт проблем химической фризики РАН, \\ 142432 Черноголовка, Московская обл., Россия \\ e-mail: zegrya@theory.ioffe.ru
}

Поступило в Редакцию 11 марта 2019 г.

В окончательной редакции 11 марта 2019 г.

Принято к публикации 25 марта 2019 г.

\begin{abstract}
Обсуждены причины разнонаправленного влияния изменений концентраций свободных носителей заряда в кристаллах кремния $p$ - и $n$-типа проводимости на поперечные размеры пор, образующихся в них в результате анодного травления в растворах плавиковой кислоты, а также влияния на размер пор плотности анодного тока. Наблюдаемые зависимости объясняются исходя из представлений об электрохимическом порообразовании в кристаллах полупроводников, как о самоорганизующихся кооперативных процессах, сопровождающихся инжекцией электронов из области химической реакции на фронте продвижения пор. Различия в размерах пор, формирующихся при одинаковых плотностях тока в кристаллах, различающихся типом и концентрациями свободных носителей заряда, связываются с эффективной температурой фронта кооперативной химической реакции на дне прорастающих пор. Эта температура, в свою очередь, коррелирует с величиной плотности мощности тепловой энергии, выделяемой в приповерхностной области травящегося кристалла либо вследствие рекомбинационных процессов, для полупроводника $p$-типа проводимости, либо процессов прямой или опосредованной передачи энергии горячих электронов колебаниям решетки в случае полупроводника $n$-типа проводимости. Проведены расчеты характерных времен релаксации инжектируемых неравновесных электронов в зависимости от концентраций основных носителей заряда в кристаллах кремния обоих типов проводимости и соответствующих им толщин областей релаксационного энерговыделения. Выявленные закономерности концентрационных изменений плотности мощности тепловыделения в прифронтальной области травящихся кристаллов кремния $p$ - и $n$-типа проводимости хорошо согласуются с наблюдаемыми изменениями размеров прорастающих в них пор.
\end{abstract}

Ключевые слова: пористый кремний, размер пор, кооперативный процесс, инжекция носителей заряда, энерговыделение.

DOI: $10.21883 /$ JTF.2019.10.48175.91-19

\section{Введение}

Образование пористых слоев в кристаллах кремния впервые было замечено в 1956 г. при изучении процессов их электрохимической полировки в растворах плавиковой кислоты [1]. Дальнейшие исследования показали, что в зависимости от условий анодирования и электрофизических характеристик исходных кристаллов кремния эти кристаллы могут быть преобразованы в макроскопически однородные пористые структуры с поперечными размерами протяженных пор от единиц нанометров до десятков микрометров. В образующихся в результате дискретного травления скелетных кристаллах с развитой внутренней поверхностью физические и химические свойства кремния претерпевают кардинальные изменения. Так, наноструктурированный пористый кремний приобретает способность люминесцировать в видимой области спектра [2], сенсибилизировать фотовозбуждение в синглетное состояние молекул адсорби- рованного на нем кислорода [3] и взрываться в контакте с окислителями, заполняющими пространство пор [4]. Способность к растворению в слабощелочных средах и биодеградации, проявляющиеся у пористого кремния с нанометровыми толщинами стенок пор [5] в сочетании с биосовместимостью, гигантской удельной поверхностью и высокой сорбционной емкостью, делают пористый кремний перспективным контейнерным материалом для создания лекарственных препаратов пролонгированного действия [6]. Благодаря необычайно широкому диапазону возможных вариаций структурных параметров пористый кремний находит все новые применения при создании самых различных устройств современной техники - от фотонных кристаллов до химических сенсоров и литиевых аккумуляторов [7].

Огромное количество выполненных к настоящему времени экспериментальных исследований, обобщению и анализу результатов которых посвящен ряд обзорных работ и монографий [8-10], свидетельствуют о том, что 
помимо задаваемых извне условий процесса электрохимического травления (состав электролита и величина анодного смещения), определяющее влияние на размерные параметры структуры образующегося пористого материала (поперечный размер пор и характерная толщина разделяющих их прослоек) оказывают тип и уровень легирования исходных кристаллов кремния.

Многочисленные попытки дать общее теоретическое толкование процессам дискретного травления кремния, однозначно связать наблюдаемые закономерности его протекания и структуру образующегося пористого материала с условиями анодирования и распределением потенциала в приграничной области исходного полупроводника не привели пока к желаемым результатам. Все обсуждавшиеся до недавнего времени модели электрохимического порообразования в полупроводниках базируются на постулате о непосредственном участии дырок валентной зоны в химическом процессе травления анодно-поляризованного в электролите кристалла. Считается, что интенсивность потока дырок к каждому из фрагментов поверхности кристалла определяет локальную скорость его травления, и, следовательно, транспорт носителей через слой объемного заряда в полупроводнике у границы с электролитом является стадией, лимитирующей скорость этого процесса. Анализ пространственного распределения анодного тока на границе раздела с электролитом в моделях, рассматривающих такую границу как непланарный барьер Шоттки $[11,12]$, а поры, как цилиндрические каналы с полусферическим днищем, показывает, что для существования устойчивой системы из параллельно прорастающих пор радиус кривизны их дна должен быть близок к толщине слоя объемного заряда, а расстояние между порами не превышать удвоенной толщины этого слоя. Для кремния $n$ - типа проводимости, в котором протекание тока через слой объемного заряда должно осуществляться за счет туннельного пробоя, наблюдаемые зависимости размерных параметров пористых слоев качественно согласуются с предсказаниями модели. При этом, однако, нужно обратить внимание на то, что однородные пористые слои образуются и в сильнолегированном вырожденном $n^{+} \mathrm{Si}$, когда под действием приложенного напряжения генерации дырок в металлизированном полупроводнике происходить уже не может, а все падение потенциала локализуется в слое Гельмгольца со стороны электролита. $\mathrm{B} p$-Si анодная поляризация должна приводить к снижению приповерхностного потенциального барьера и к последующему выпрямлению зон (благодаря гидрированию поверхности кремния в контакте с HF-содержащими электролитами не возникает собственных электрически активных поверхностных состояний и пиннинга уровня Ферми в запрещенной зоне не происходит). Поэтому, согласно модели, с ростом уровня легирования или приложенного напряжения (плотности тока) следовало бы ожидать уменьшения размеров пор, что противоречит экспериментальным наблюдениям.
Очевидно, что на протекание анодного тока не могут не оказывать влияния механизм и кинетика происходящих электрохимических реакций, и игнорирующие их упрощенные модели не в состоянии описать формирование пор во всем диапазоне изменений условий анодного процесса.

Своеобразная попытка учета гетерогенной кинетики сделана Г. Фелем и другими исследователями, которые, основываясь на наблюдениях осцилляций анодного тока (или потенциала), возникающих в стационарных режимах порообразования, приходят к гипотезе об импульсном характере процессов анодного травления. Такой эффект, без достаточно серьезного химического обоснования, приписывается в этих работах периодически возникающей пассивации фронта травления пленками анодных окислов, растворяющихся затем в исходном электролите [13].

В рамках имеющихся представлений крайне сложно объяснить наблюдаемые структурные изменения в слоях пористого кремния, допускающие вариации размеров пор на несколько порядков величины при изменении типа и уровня легирования исходных кристаллов, анодируемых в одинаковых условиях. Иной и, возможно, более продуктивный подход к объяснению формирования структур пористых кристаллов в процессах электрохимического травления полупроводников, может предложить развиваемая нами теория анодного порообразования, представляющая его как кооперативный химический процесс, инициируемый падением потенциала на границе раздела полупроводник-электролит $[14,15]$.

\section{1. Причины зависимости размеров пор в пористом кремнии от типа проводимости и концентрации свободных носителей заряда в исходном кристалле}

Непосредственной целью настоящей работы является объяснение причины сильного и противоположного по своему характеру влияния изменений концентраций свободных носителей заряда в кристаллах кремния $p$ и $n$-типа проводимости на поперечный размер каналов пор, распространяющихся в них в стационарных режимах анодного травления. Сразу отметим, что поставленная задача не имеет прямого отношения к вопросу о зародышеобразовании пор и размерах их входных отверстий на поверхности кристалла [15]. Речь пойдет об установившихся процессах, протекающих при достаточных отклонениях от пороговых значений напряжения начала порообразования, обеспечивающих устойчивое распространение пор в кремнии. Не будут также затрагиваться процессы образования крупных макропор в слаболегированных кристаллах кремния, развивающиеся в условиях лавинного пробоя, при которых анодная реакция может протекать с участием некооперативного 


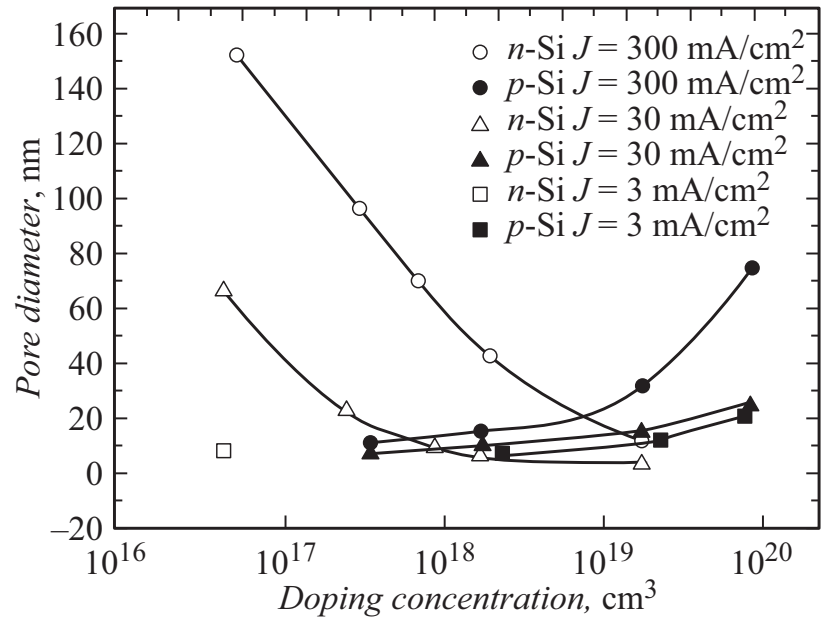

Pис. 1. Экспериментальные зависимости размера пор в кристаллах $n$ - и $p$-Si от концентрации равновесных носителей заряда для трех значений плотности анодного тока. Данные взяты из работы [12].

механизма, характерного для процесса сплошного травления кремния [15].

Экспериментально полученные зависимости размеров пор в кремнии обоих типов проводимости от уровня легирования для различных плотностей анодного тока в HF-этанольном электролите приведены в работе Лемана [12] и представлены на рис. 1.

Как видно, для кремния $p$-типа проводимости при уровне дырочной концентрации $\sim 10^{17} \mathrm{~cm}^{-3}$ размер пор мал (менее $10 \mathrm{~nm})$ и слабо зависит от плотности тока, тогда как для $n$-Si при такой же электронной концентрации размер пор возрастает с увеличением плотности тока $j$ от тех же $\sim 10 \mathrm{~nm}$ при $j=3 \mathrm{~mA} / \mathrm{cm}^{2}$, до $\sim 140 \mathrm{~nm}$ при $j=300 \mathrm{~mA} / \mathrm{cm}^{2}$. С ростом концентраций свободных носителей заряда и фиксированном значении $j$ размер пор в $n$-Si падает, тогда как в $p$-Si возрастает. При этом для концентраций, больших $10^{19} \mathrm{~cm}^{-3}$, темп увеличения размеров пор с возрастанием плотности тока для $p-\mathrm{Si}$ становится выше, чем для $n$-Si.

Согласно нашим представлениям, в основе самоорганизующихся анодных процессов, приводящих к появлению и прорастанию пор в алмазоподобных полупроводниковых кристаллах, лежат кооперативные химические реакции нуклеофильного замещения, протекающие с участием компактных групп хемосорбированных анионов, образующих плотные адсорбционные покрытия, и координационно-насыщенных атомов приповерхностного слоя решетки. Эти реакции инициируются при достижении определенной для данной комбинации составов полупроводника и хемосорбированных анионов величины падения потенциала в слое Гельмгольца, обеспечивающей возможность туннелирования электронов с заполненных несвязывающих орбиталей адатомов на вакантные состояния антисвязывающих или несвязывающих орбиталей атомов приповерхностного слоя кристалла $[14,15]$. Разрыв химических связей в кристалле при когерентной релаксации образовавшихся переходных комплексов и отделении первичных полимерных продуктов кооперативной реакции сопровождается импульсным высвобождением электронов в зону проводимости, что и обеспечивают протекание анодного тока. Становящиеся вакантными связи атомов на вновь образовавшейся поверхности кристалла и отделяющихся от него продуктах заполняются поступающимися из раствора анионами. Этим завершается единичный цикл процессов, обеспечивающих пошаговое продвижение канала поры вглубь кристалла.

В случае кремния в формировании локальных группировок из связанных шестикоординированных переходных комплексов, возникающих при синхронном переносе адсорбированных анионов фтора с атомов поверхности кристалла на атомы его приповерхностного слоя, участвуют их несвязывающие $d^{2} s p^{3}$-гибридные орбитали [15]. В результате релаксации систем таких комплексов с возвращением атомов кремния в базовое $s p^{3}$-гибридное состояние, электроны, формировавшие химические связи с атомами нижележащего слоя решетки, оказываются на энергетических уровнях несвязывающих орбиталей, соответствующих зоне проводимости, и оказываются свободными. Двумерная плотность электронов $\left(\rho_{s}\right)$, высвобождаемых в пятне кооперативной реакции в момент отделения от кристалла ее первичных продуктов - полимерных цепочек $\left(\mathrm{SiF}_{2}\right)_{n}$, должна составлять $2.71 \cdot 10^{15} \mathrm{~cm}^{-2}$ в плоскости (100). Как минимум половина из появившихся свободных электронов немедленно выносится электрическим полем в объем кристалла. Оставшиеся переходят на вакантные состояния оборванных связей атомов вновь образованной поверхности кремния, что предполагает рассеивание на этой поверхности их избыточной энергии и передачу импульса фононам. Заметим, что доля энергии, способной выделиться на фронте анодной реакции благодаря последнему процессу, не должна зависеть от электрофизических параметров травящегося кристалла. Электроны, перешедшие на орбитали оборванных связей атомов обновленной поверхности, могут фиксироваться на них до момента последующей химической адсорбции анионов фтора. Но в любом случае все высвобождаемые при отщеплении продуктов кооперативной реакции электроны переходят в кристалл в интервале времени между ее последовательными актами, давая этим свой вклад в ток цепи ячейки.

В прямозонных полупроводниковых кристаллах со структурой сфалерита аналогичные процессы осуществляются с участием антисвязывающих $s p^{3 *}$-гибридных орбиталей, чем и обусловливается различие доминирующих кристаллографических направлений распространения пор в кремнии - $\langle 100\rangle$ и в кристаллах $\mathrm{A}^{3} \mathrm{~B}^{5}-$ $\langle 111\rangle[14]$.

Поскольку каждый элементарный акт кооперативной химической реакции продвигает фронт распространения индивидуальной поры в кремнии на полпериода решетки 
вдоль одного из кристаллографических направлений типа $\langle 100\rangle$, т.е. на $0.2715 \mathrm{~nm}$, то по скорости прорастания пор можно оценить частоту повторений элементарных актов травления и порождаемых ими импульсов высвобождения электронов. Так, в режимах, чаще всего использующихся при получении слоев мезопористого кремния, нормальные скорости прорастания слабоветвящихся пор в кремниевых пластинах с кристаллографической ориентацией поверхности (100) составляют $3-5 \mu \mathrm{m} / \mathrm{min}$. Соответствующая этим значениям скоростей оценка, по минимуму, частот генерации пакетов электронов у дна каждой из продвигающихся пор дает 190-310 Hz.

В полупроводниках $p$-типа проводимости инжектируемые с поверхности электроны должны высвобождать свою потенциальную энергию, рекомбинируя с равновесными дырками в непосредственной близости от фронта химической реакции, а в кристаллах $n$-типа проводимости - напрямую рассеивать на колебаниях решетки избыточную кинетическую энергию, приобретаемую ими в результате прохождения градиента потенциала в приповерхностном слое объемного заряда. Последний эффект существенен для невырожденных умеренно легированных кристаллов $n$-типа проводимости. При поляризации в электролите сильнолегированного $n$-полупроводника, вырожденного во всем объеме, включая поверхностные слои, практически все падение потенциала локализуется в слое Гельмгольца, и генерируемые в ходе анодной реакции электроны уже не получают дополнительной кинетической энергии, перемещаясь в объем кристалла.

Следствием энерговыделения, сопровождающего как рекомбинационные, так и релаксационные процессы, становится разогрев прифронтальной области кристалла. Поскольку кремний является непрямозонным полупроводником, то как в $p$ - так и $n$-кристаллах практически вся энергия, привносимая инжектируемыми неравновесными электронами, должна переходить в тепловую энергию колебаний решетки. С повышением локальной температуры в зоне реакции возрастают амплитуды и частоты тепловых флуктуаций, способствующих преодолению активационных барьеров для запуска многочастичных кооперативных процессов нуклеофильной атаки адсорбированных анионов на атомы кристалла на дне каждой из прорастающих пор. Это, в свою очередь, создает условия для пространственного расширения локальных областей протекания кооперативной реакции и увеличения частоты повторения ее дискретных актов при данной, фиксированной величине падения потенциала в адсорбционном слое. Как следствие, должно происходить увеличение плотности и размеров пор с увеличением глубины их проникновения в кристалл, сопровождающееся возрастанием анодного тока, что и наблюдается на практике. В этом смысле можно говорить о самоактивации анодного травления при углублении фронта порообразования в объем кристалла. Если же процесс проводится в гальваностатическом режиме, т.е. при неизменной величине интегральной плотности тока и соответственно удельной массовой скорости растворения кремния, увеличение поперечных размеров пор должно вызывать снижение скорости их прорастания вглубь кристалла.

Эффективная температура в области реакции $\left(T_{\text {eff }}\right)$ и соответственно степень проявления описанных эффектов должны определяться величиной плотности мощности тепловой энергии $(M)$, выделяемой у фронта продвижения пор, а также скоростью отведения тепла во внешнюю среду. Очевидно, что вклад инжектируемых в кристалл электронов в плотность мощности, выделяющейся в ходе электрохимической реакции энергии, должен быть, с одной стороны, пропорционален ее удельной скорости и соответственно плотности анодного тока $(j)$, и, с другой стороны - обратно пропорционален толщине прифронтальной области кристалла, в которой происходит рассеивание энергии электронов $(L)$. То есть при установившемся режиме теплоотвода $T_{\text {eff }} \sim M \sim j / L$. В конечном счете, поперечный размер прорастающих пор должен изменяться симбатно изменениям $j / L$.

\section{1. Размеры областей энерговыделения, сопутствующего прорастанию пор в кристаллах кремния $p$-типа проводимости}

Рассмотрим теперь подробнее процессы диссипации энергии, связанные с рассеянием в аноднополяризованных кристаллах кремния неравновесных электронов, инжектируемых в них с поверхности, из области гетерогенной химической реакции.

В кристаллах $p$-типа проводимости глубина проникновения инжектируемых электронов определяется их диффузионной длиной:

$$
L_{D}=\left(D_{n} \tau_{l}\right)^{1 / 2}
$$

где $D_{n}-$ коэффициент диффузии для электронов, составляющий в кремнии при $300 \mathrm{~K}$ величину $D_{n}=36 \mathrm{~cm}^{2} / \mathrm{s}[16], \tau_{l}$ - время жизни электронов. Это время определяется двумя каналами рекомбинации излучательной и безызлучательной (оже-) рекомбинацией:

$$
\frac{1}{\tau_{l}}=\frac{1}{\tau_{p h}}+\frac{1}{\tau_{A}}
$$

где $\tau_{p h}$ - время излучательной рекомбинации, $\tau_{A}-$ время оже-рекомбинации.

В нашем случае начальная мгновенная концентрация неравновесных электронов, инжектируемых из пятна реакции, может соответствовать концентрациям электронов в металле, $n \sim 10^{22} \mathrm{~cm}^{-3}$, что много выше достижимых концентраций равновесных дырок. В этих условиях интенсивность процессов оже-рекомбинации должна значительно превышать интенсивность излучательной 
рекомбинации. Скорость излучательной рекомбинации можно представить в виде

$$
R_{p h}=B n p,
$$

где $B-$ коэффициент излучательной рекомбинации, который для кремния хорошо известен и равен $B=1.1 \cdot 10^{-14} \mathrm{~cm}^{3} / s[16] ; n$ и $p-$ концентрации электронов и дырок соответственно.

В кремнии также возможны два процесса оже-рекомбинации. Оже-рекомбинация с возбуждением электрона, так называемый CHCC-процесс. Скорость такого процесса равна

$$
R_{A}^{C}=C^{C} n^{2} p,
$$

где $C^{C}$ - коэффициент оже-рекомбинации для CHCCпроцесса. Другой процесс - это оже-рекомбинация с возбуждением дырки, так называемый CHHS-процесс. Для такого процесса скорость оже-рекомбинации равна

$$
R_{A}^{H}=C^{H} n p^{2} .
$$

Здесь $C^{H}-$ коэффициент оже-рекомбинации для CHHS-процесса. Для кремния при $T=300 \mathrm{~K}, C^{C}=$ $=1.1 \cdot 10^{-30} \mathrm{~cm}^{6} / \mathrm{s}$ и $C^{H}=0.3 \cdot 10^{-30} \mathrm{~cm}^{6} / \mathrm{s}[16]$. Зная скорости рекомбинации неравновесных носителей, можно найти время их жизни. Для этого воспользуемся уравнением непрерывности для электронов

$$
\frac{\partial n}{\partial t}=-R_{u}-R_{A}=-B n p-C^{C} n^{2} p-C^{H} n p^{2} \equiv-\frac{n}{\tau_{l}} .
$$

Тогда

$$
\frac{1}{\tau_{l}}=B p+C^{C} n p+C^{H} p^{2} ; \quad R_{A}=R_{A}^{C}+R_{A}^{H} .
$$

Подставляя выражение для $\tau_{l}$ из (7) в выражение (1), получим, что

$$
L_{D}=\left[\frac{D}{\left(B+C^{C} n+C^{H} p\right) p}\right]^{1 / 2} .
$$

Здесь $n$ и $p$ - исходные концентрации электронов и дырок на момент начала рекомбинации. Значение $n$ принимается одинаковым и равным $1 \cdot 10^{22} \mathrm{~cm}^{-3}$.

На рис. 2 представлена полученная зависимость диффузионной длины инжектированных электронов от концентрации равновесных дырок $p$. Видно, что глубина проникновения неравновесных электронов, появляющихся в приповерхностном слое, существенно зависит от концентрации равновесных дырок. Так как для рассматриваемых процессов всегда выполняется условие $(p \ll n)$, то, как следует из $(8)$, глубина проникновения $L_{D}$ убывает с ростом $p$ как $L_{D} \propto p^{-1 / 2}$.

Итак, вследствие интенсивной оже-рекомбинации в кристаллах кремния с достаточно высокой дырочной концентрацией, $p>10^{18} \mathrm{~cm}^{-8}$, энергия электронов, высвобождаемых на фронте порообразования, должна выделяться в тонком слое, толщиной порядка или меньше

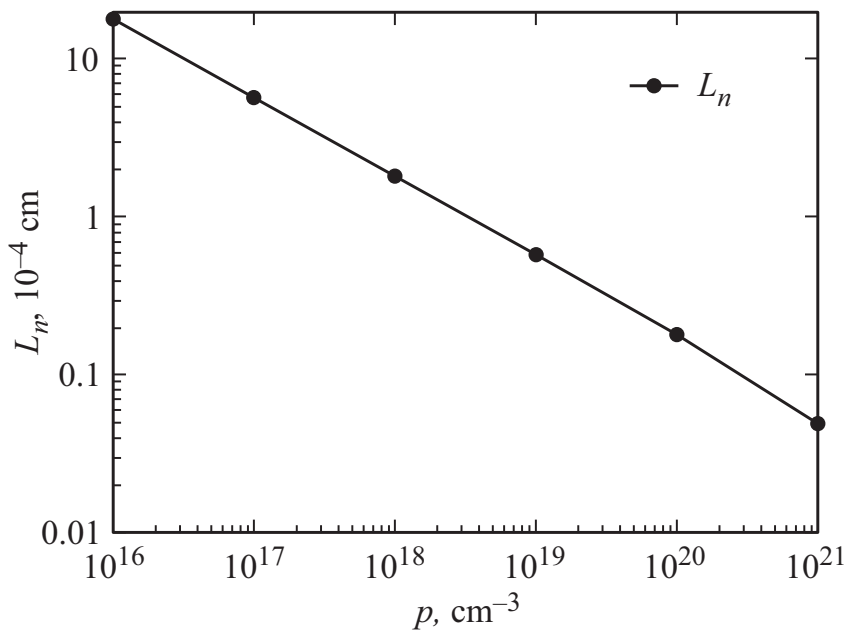

Рис. 2. Зависимость диффузионной длины инжектированных электронов от концентрации равновесных дырок $p$.

одного микрона (рис. 2); при уменьшении концентрации дырок область рекомбинации существенно расширяется, достигая десятков $\mu$ m при $n \sim 10^{16} \mathrm{~cm}^{-3}$.

Поскольку в единичном акте электронно-дырочной рекомбинации выделяется энергия порядка $E_{g}=1.1 \mathrm{eV}$, то количество энергии, высвобождаемой в кристалле между последовательными актами отщепления продуктов реакции и выброса электронов в пересчете на $1 \mathrm{~cm}^{2}$ реакционной поверхности, должно составить $\rho_{s} E_{g}=2.98 \cdot 10^{15} \mathrm{eV} / \mathrm{cm}^{2}$ или $4.78 \cdot 10^{-4} \mathrm{~J} / \mathrm{cm}^{2}$. Полагая, в частности, что элементарные акты кооперативной реакции, обеспечивающей прорастание пор, повторяются с частотой $v=250 \mathrm{~Hz}$ (соответствует скорости продвижения фронта неветвящихся пор $\sim 4 \mu \mathrm{m}$ ), то за секунду на $1 \mathrm{~cm}^{2}$ суммарной площади реакционной поверхности пор должно выделиться $\sim 0.12 \mathrm{~J}$. В расчете на единицу исходной поверхности кристалла тепловыделение должно быть ниже, пропорционально степени пористости формирующегося слоя. Для сравнения при анодировании сильнолегированных пластин $p$-Si в $\mathrm{HF}$-этанольном электролите плотность анодного тока $\sim 0.1 \mathrm{~A} / \mathrm{cm}^{2}$ (скорость роста слоя $\sim 4 \mu / \mathrm{min}$ и пористость 75-80\%) достигается при падении потенциала на электрохимической ячейке $\sim 3 \mathrm{~V}$, т. е. общее количество выделяемого в ней тепла в пересчете на $1 \mathrm{~cm}^{2}$ травящейся поверхности кремния близко к $0.3 \mathrm{~J} / \mathrm{cm}^{2} \mathrm{~s}$. Таким образом, как и следовало ожидать, тепло, генерируемое в кристалле кремния вследствие анодной реакции, составляет лишь меньшую часть тепла, выделяемого при прохождении тока в цепи ячейки. Оценим теперь удельную плотность мощности тепловыделения $(M)$ за счет рекомбинационных процессов в приповерхностном слое кремния толщиной $L_{D}$ (число рекомбинирующих на $L_{D}$ электронов составляет 0.632 от числа инжектированных электронов). В рассматриваемом примеpe, при скорости тепловыделения на фронте реакции $0.12 \mathrm{~J} / \mathrm{cm}^{2} \mathrm{~s}$ и пористости слоя $\sim 80 \%$, если принять 
$L_{D}=1 \mu \mathrm{m}$ (что, согласно рис. 2 , должно обеспечиваться дырочной концентрацией $\sim 3 \cdot 10^{18} \mathrm{~cm}^{2}$ ), то объемная плотность мощности составит $M \sim 600 \mathrm{~J} / \mathrm{cm}^{3} \mathrm{~s}$. Такой удельной мощности достаточно, чтобы в отсутствие теплоотвода нагреть за $1 \mathrm{~s}$ кристалл кремния объемом $1 \mathrm{~cm}^{3}$ на $\sim 360^{\circ} \mathrm{C}$. Очевидно, что неизбежно возникающий разогрев реакционной поверхности в процессе порообразования, усиливающийся с повышением уровня легирования кристаллов $p$-Si, должен становиться причиной интенсификации кооперативной химической реакции и пространственного расширения областей ее протекания, а соответственно и поперечного размера пор.

Нами, в частности, было проведено сравнение параметров структуры пористого кремния, полученного в диапазоне плотностей тока $35-100 \mathrm{~mA} / \mathrm{cm}^{2}$ в этанольном растворе плавиковой кислоты 1:1 на пластинах сильнолегированного $p$-Si $\langle 100\rangle$ с концентрациями носителей заряда $1.1 \cdot 10^{10} \mathrm{~cm}^{-3}$ и $2.4 \cdot 10^{19} \mathrm{~cm}^{-3}$. Для отделенных от подложек пористых слоев методами адсорбционно-структурного анализа [17] по изотермам адсорбции и десорбции азота при $77 \mathrm{~K}$ на анализаторе ASAP 2020 (Micromeritics) были определены средний диаметр пор $\left(d_{S}\right)$; удельная площадь поверхности $\left(S_{S}\right)$; суммарный удельный объем пор $\left(V_{\sum}\right)$ и рассчитана степень пористости $(P)$. Графики зависимостей полученных величин от плотности анодного тока приведены на рис. 3.

Как видно, поперечный размер пор и остальные связанные с ним параметры структуры пористых слоев монотонно возрастают с повышением плотности анодного тока, а увеличение дырочной концентрации в исходном кристалле в 2.2 раза сопровождается расширением пор в $1.1-1.3$ раза.

\section{2. Пространственное распределение тепловой энергии, рассеиваемой инжектированными из области анодной реакции электронами в кристаллах $n$-типа проводимости}

При анодной поляризации невырожденного полупроводника $n$-типа проводимости требуемое для начала его травления падение потенциала в слое Гельмгольца (точнее, между слоем хемосорбированных анионов и приповерхностным слоем атомов кристалла) достигается после выполнения условия поверхностного вырождения [14], т.е. при изгибе зон у границы раздела с электролитом, близком к ширине запрещенной зоны $\left(E_{g}\right)$. Поэтому свободные электроны, появляющиеся в ходе релаксации переходных комплексов химической реакции на поверхности кристалла, приобретают избыточную по отношению к состоянию свободных электронов в объеме полупроводника энергию, практически равную $E_{g}$, и в этом смысле являются горячими. Плотность тепловой энергии, выделяющейся тогда вблизи фронта химической реакции, должна определяться преобладающим механизмом релаксации (термолизации) в кристалле инжектируемых горячих электронов, который в свою очередь кардинальным образом зависит от равновесной концентрации электронов в полупроводнике $n$-типа. При высокой концентрации электронного газа в полупроводнике основной вклад в рассеяние избыточной энергии инжектируемых горячих электронов должно вносить электрон- электронное взаимодействие, затрагивающее весь объем кристалла. В этом случае, плотность выделяемой тепловой энергии, определяющей температуру фронта реакции, окажется минимальной.

Конкурирующим с электрон-электронным взаимодействием и доминирующим над ним в слабо- и умеренно-легированных кристаллах является электрон-фононное взаимодействие. При электрон-фононном взаимодействии возможны три процесса рассеяния энергии горячих электронов: а) индуцированное испускание фононов; его вероятность пропорциональна числу фононов; б) поглощение фононов; вероятность поглощения также пропорциональна числу фононов; в) спонтанное излучение фононов.

Вероятность рассеяния в борновском приближении можно вычислить согласно выражению [18]:

$$
W_{i \rightarrow f}=\frac{2 \pi}{\eta}|\langle f|t|\rangle|^{2} \delta\left(\varepsilon_{i}-\varepsilon_{f}\right),
$$

где $|i\rangle$ - начальное состояния, $|f\rangle$ - конечное, $\varepsilon_{i}, \varepsilon_{f}-$ энергии начальных и конечных состояний электронов; $T$ - оператор квантовых переходов.

При электрон-электронном рассеянии состояния $|i\rangle$ и $|f\rangle$ - состояния взаимодействующих электронов проводимости. В случае рассеяния на фононах $|i\rangle$ и $|f\rangle$ - эти состояния для системы: один электрон плюс фонон; для однофононных процессов $T$ - это оператор электрон-фононного взаимодействия.

1.2.1. Рассеяние энергии горячих электронов на фононах. Хотя в кремнии закон дисперсии для электронов анизотропен, т.е. характеристика рассеяния зависит не только от энергии электрона, но и от направления его импульса $h \mathbf{k}$ и импульса фонона $h \mathbf{q}$, для оценки эффекта мы не будем учитывать анизотропию ни фононного спектра, ни электронного; будем считать, что фононы с любым волновым вектором q разделяются на продольные (акустические и оптические) и поперечные (акустические и оптические). Тогда для вероятности перехода получаем [19]

$W_{\mathbf{k} \rightarrow \mathbf{k}^{\prime}}^{ \pm s \mathbf{q}}=\frac{2 \pi}{h}\left(M_{\mathbf{k} \rightarrow \mathbf{k}^{\prime}}^{ \pm s \mathbf{q}}\right)^{2}\left(N_{s \mathbf{q}}+\frac{1}{2} \pm \frac{1}{2}\right) \delta\left(\varepsilon_{\mathbf{k}}-\varepsilon_{\mathbf{k}^{\prime}} n h \omega_{s \mathbf{q}}\right)$.

Здесь $\mathbf{k}$ - начальное состояние электрона, $\mathbf{k}^{\prime}-$ конечное; верхний знак относится к переходу с испусканием фонона $s \mathbf{q}$, нижний - к переходу с поглощением фонона, $s$ - нумерует различные ветви фононного спектра (акустические, оптические, продольные, поперечные); $N_{s q}$ - числа заполнения фононов в каждой 

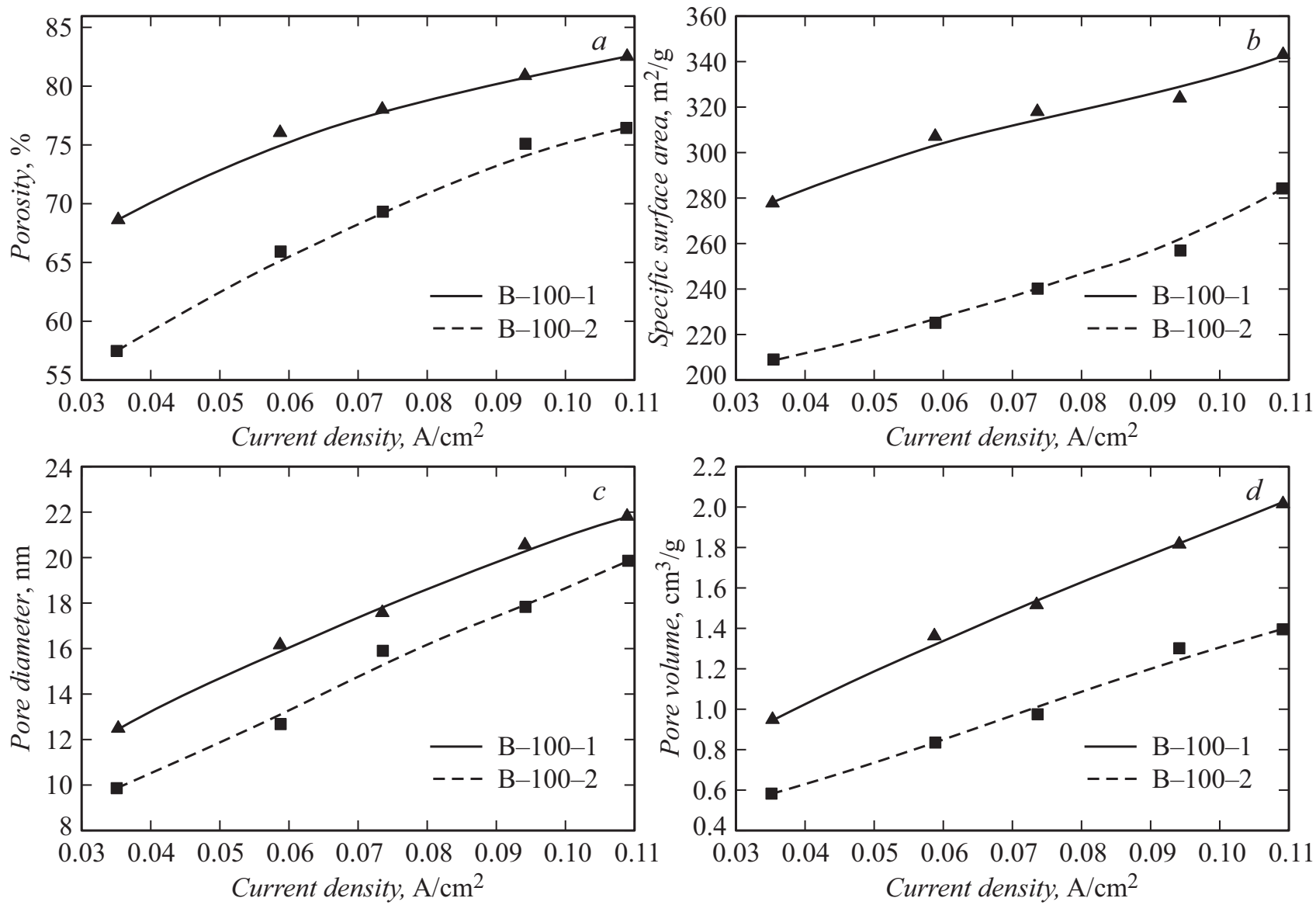

Рис. 3. Зависимости от величины плотности анодного тока степени пористости $(a)$, удельной площади поверхности $(b)$, среднего диаметра пор $(c)$ и их удельного суммарного объема $(d)$ для структур пористого кремния, полученных из сильнолегированных кристаллов кремния $p$-типа проводимости с уровнями легирования $p=2.4 \cdot 10^{19} \mathrm{~cm}^{-3}$ (образцы $\left.\mathrm{B}-100-1\right)$ и $p=1.1 \cdot 10^{19} \mathrm{~cm}^{-3}$ (образцы В-100-2).

фононной моде; $M_{\mathbf{k} \rightarrow \mathbf{k}^{\prime}}^{ \pm s \mathbf{q}}-$ матричный элемент перехода. Дельта-функция выражает закон сохранения энергии электронов.

Следует заметить, что в нашем случае речь идет о релаксации горячих электронов, тогда когда их энергия $\varepsilon \gg k_{B} T$. Для такого случая вероятность поглощения фононов электронами экспоненциально мала по сравнению с вероятностью испускания фононов.

Будем считать, что матричный элемент в (10) не зависит от $\mathbf{k}$ и $\mathbf{k}^{\prime}$ порознь, а зависит только от $\mathbf{q}= \pm\left(\mathbf{k}-\mathbf{k}^{\prime}\right)$. Поэтому в рамках такого приближения [19]

$$
\left|M_{\mathbf{k} \rightarrow \mathbf{k}^{\prime}}^{ \pm s \mathbf{q}}\right|^{2}=\frac{1}{L^{3}} B(q) \delta_{\mathbf{k}^{\prime}, \mathbf{k}, \mathbf{q}}
$$

где $B-$ зависит только от модуля $q ; L^{3}-$ нормировочный объем.

В дальнейшем мы рассмотрим рассеяние энергии электронов на оптических фононах (речь идет о деформационном рассеяние ); рассеянием на акустических фононах мы пренебрегаем в силу его малости. Для этого случая

$$
B(q)=\eta^{2} \Gamma^{2} / 2 \bar{\rho} \omega_{0},
$$

где $\Gamma$ - константа деформационного потенциала оптических фононов, $\omega_{0}$ - предельная частота оптического фонона, $\bar{\rho}$ - плотность приведенной массы.

Тогда для частоты испускания фонона получаем [19]

$$
\frac{1}{\tau_{\varepsilon}}=\frac{1}{\tau_{D O}}\left(N_{0}+1\right)\left(\frac{\varepsilon-\eta \omega_{0}}{\eta \omega_{0}}\right)^{1 / 2} .
$$

Здесь $N_{0}=\left(e^{\frac{\eta \omega_{0}}{k_{B} T}}-1\right)^{-1} ; \varepsilon-$ энергия неравновесного электрона; $\frac{1}{\tau_{D O}}=\frac{m_{d} \Gamma^{2} p_{0}}{2 \pi \eta^{2}} \bar{\rho} \omega_{0} ; p_{0}=\left(2 m_{d} \eta \omega_{0}\right)^{1 / 2} / \eta ; \omega_{0}-$ предельная частота оптического фонона; $m_{d}$ - эффективная масса плотности состояний.

Из полученного выражения для $\frac{1}{\tau_{\varepsilon}}$ следует, что время рассеяния горячего электрона на фононах не зависит от концентрации равновесных электронов $n$. Подставляя в (13) параметры кремния [16]: $\varepsilon=1.12 \mathrm{eV}$, $\omega_{0}=0.063 \mathrm{eV}, m_{d}=0.567 m_{0}$ (где $m_{0}$ - масса свободного электрона), $\rho=2.33 \mathrm{~g} / \mathrm{cm}^{3}, \Gamma=e^{2} / a_{0}=4.2 \cdot 10^{4} \mathrm{eV}$ (где $a_{0}$ - параметр решетки и равен $5.431 \AA$ ), получаем что $\frac{1}{\tau_{\varepsilon}} \approx 2.65 \cdot{ }^{12} \mathrm{~s}^{-1}$. 
Таким образом, если рассеяние на фононах остается единственным механизмом передачи решетке энергии горячих электронов, то практически вся их энергия будет затрачиваться на разогрев очень узкой области кристалла, примыкающей к продвигающемуся фронту электрохимической реакции. Толщина такого слоя

$$
L_{p}=\tau_{\varepsilon} v,
$$

где скорость $v=\left(2 \varepsilon / m_{c}\right)^{1 / 2}, m_{c}$ - масса проводимости кремния и $m_{c}=0.26 m_{0}$. Тогда для скорости получаем $v=1.23 \cdot 10^{8} \mathrm{~cm} / \mathrm{s}$. Подставляя в (14) это значение для скорости, для $\frac{1}{\tau_{\varepsilon}} \approx 1.65 \cdot 10^{12} \mathrm{~s}^{-1}$, получаем $L_{P}=43.6 \cdot 10^{-1} \mathrm{~cm}$.

\subsection{2. Рассеяние горячих электронов на рав-} новесном электронном газе. Если инжектируемый горячий электрон с импульсом $h \mathbf{k}$ рассеивается на распределении равновесных электронов с функцией распределения $f_{p}$ (концентрацией $n$ ), то вероятность рассеяния электрона из состояния $\mathbf{k}$ в $\mathbf{k}^{\prime}$ равна [19]

$$
\begin{aligned}
W_{\mathbf{k}-\mathbf{k}^{\prime}}= & \sum_{\mathbf{p}, \mathbf{p}^{\prime}} f_{\mathbf{p}}\left(1-f_{\mathbf{p}^{\prime}}\right) \frac{2 \pi}{h}\left|M_{\mathbf{k}, \mathbf{p} \rightarrow \mathbf{k}^{\prime}, \mathbf{p}^{\prime}}\right|^{2}\left(N_{s \mathbf{q}}+\frac{1}{2} \pm \frac{1}{2}\right) \\
& \times \delta\left(\varepsilon_{\mathbf{k}}+\varepsilon_{\mathbf{p}}-\varepsilon_{\mathbf{k}^{\prime}}-\varepsilon_{\mathbf{p}^{\prime}}\right) .
\end{aligned}
$$

Здесь $M_{\mathbf{k}, \mathbf{p} \rightarrow \mathbf{k}^{\prime}, \mathbf{p}^{\prime}}-$ матричный элемент электронэлектронного взаимодействия; $\varepsilon_{\mathbf{k}}, \varepsilon_{\mathbf{p}}-$ энергии электронов в начальном состоянии; $\varepsilon_{\mathbf{k}^{\prime}}, \varepsilon_{\mathbf{p}^{\prime}}-$ энергии электронов в конечном состоянии.

Если пренебречь экранированием носителями, то вероятность рассеяния можно вычислить в явном виде [19]

$$
W_{\mathbf{k}-\mathbf{k}^{\prime}} \approx \frac{1}{L^{3}} \frac{m e^{4}}{h^{2} \chi^{2}}(2 \pi m T) \frac{1}{q^{5}} \exp \left[-\frac{\varepsilon_{\mathbf{k}^{\prime}}}{T}+\frac{k^{2}}{2 m T}\right] .
$$

Здесь $k^{2}$ - общая проекция $\mathbf{k}$ и $\mathbf{k}^{\prime}$ на плоскость, перпендикулярную q; q - переданный импульс при кулоновском взаимодействии; $\chi$ - диэлектрическая проницаемость кристалла. В результате для вероятности рассеяния энергии получаем

$$
\frac{1}{\tau_{e e}}=8 \pi \Lambda\left(\frac{E_{B}}{\eta}\right) n k^{-3},
$$

где $E_{B}=\frac{m_{d} e^{4}}{2 \eta^{2} \chi^{2}}-$ боровская энергия электрона, $k=\left(2 m_{d} \varepsilon\right)^{1 / 2} / \eta, \varepsilon-$ энергия инжектируемого электрона; $n-$ концентрация равновесных электронов; $\Lambda$ кулоновский логарифм.

Если подставить в (17) параметры кремния [16]: $\varepsilon=1.12 \mathrm{eV}, m_{d}=0.567 m_{0}, \chi=11.7, \Lambda=15$, то получаем зависимость характеристического времени рассеяния горячего электрона от концентрации равновесных электронов (рис. 4). Как видно, время рассеяния на электронах становится сопоставимо со временем рассеяния на фононах $\left(\frac{1}{\tau_{\varepsilon}} \approx 2.45 \cdot 10^{12} \mathrm{~s}^{-1}\right)$ при концентрации равновесных электронов $n=6 \cdot 10^{18} \mathrm{~cm}^{-3}$.

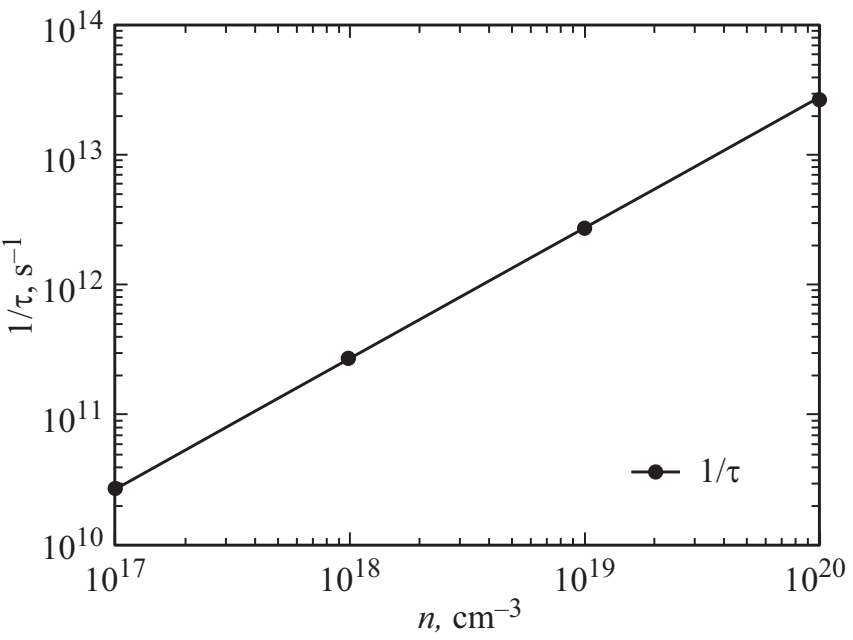

Рис. 4. Зависимость частоты элементарных актов рассеяния энергии неравновесных электронов на электронном газе.

Поскольку энерговыделение при прямом взаимодействии горячих электронов с решеткой происходит в очень узкой области кристалла, примыкающей к фронту продвижения пор (доли $\mu \mathrm{m})$, а энергия горячих электронов, передаваемая электронам проводимости, рассеивается по всему объему кристалла (толщиной, как правило, сотни $\mu \mathrm{m})$, то плотность мощности тепловой энергии, выделяемой вблизи реакционной поверхности и определяющей эффективную температуру процесса, должна заметно снижаться с повышением электронной концентрации $n$ выше $10^{17} \mathrm{~cm}^{-3}$. Когда же электронная концентрация в полупроводнике начинает превышать порог вырождения (для кремния это выше $10^{19} \mathrm{~cm}^{-3}$ ), и приповерхностный слой объемного заряда перестает существовать, исчезает и причина дополнительного тепловыделения при продвижении пор. Поэтому размеры пор в сильнолегированном $n^{+}$-Si принимают минимальные при заданных плотностях тока значения.

На рис. 5 приведены рассчитанные в зависимости от уровня концентраций свободных носителей заряда в кристаллах кремния $p$ - и $n$-типа проводимости, значения обратных величин протяженностей областей рассеивания энергии электронов, инжектируемых в них с фронта протекания кооперативной реакции. Для $n-\mathrm{Si}$, не прибегая к громоздким вычислениям, можно привести лишь три значения $1 / L$. Первое $\sim 2.17 \cdot 10^{4} \mathrm{~cm}^{-1}$, относящееся к рассеянию энергии горячих электронов на фононах без посредничества электронов проводимости, что справедливо для $n<10^{17} \mathrm{~cm}^{-3}$; второе соответствующее равенству времен рассеяния энергии на фононах и на электронном газе, занимающем весь объем кристалла, что предполагает практически двукратное уменьшение плотности энерговыделения у фронта реакции и эквивалентно удвоению толщины области рассеяния $\left(n \sim 6 \cdot 10^{18} \mathrm{~cm}^{-3}\right)$; и третье - предполагающее полную передачу избыточной энергии инжектируемых электронов электронному газу травящегося кристалла 


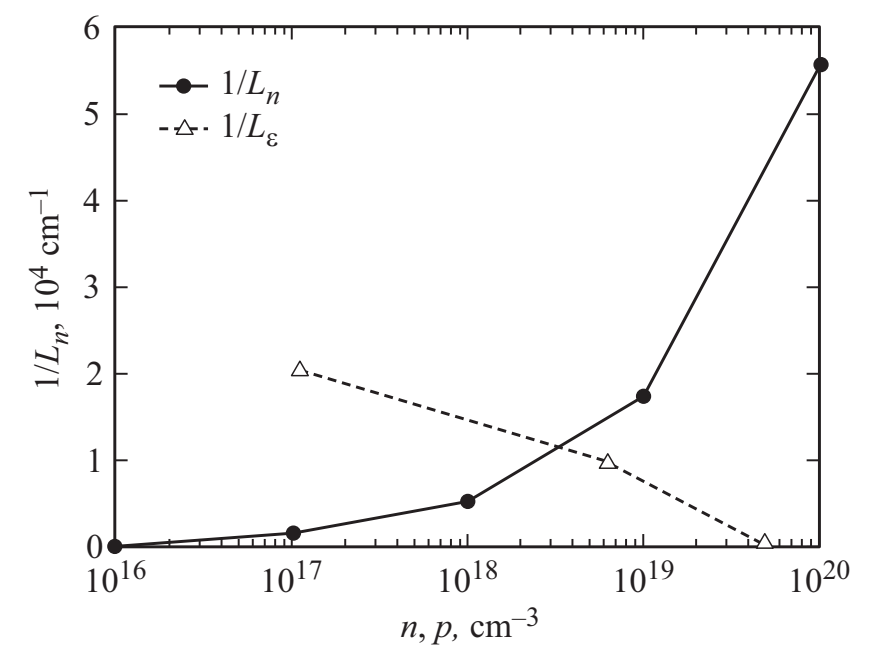

Pис. 5. Зависимости обратной диффузионной длины для инжектируемых электронов от концентрации дырок $(p)$ в кристаллах кремния $p$-типа проводимости и обратной величины эффективной протяженности области рассеяния их энергии от концентрации равновесных электронов $(n)$ в кристаллах $n$-типа.

толщиной $h$. В последнем случае, реализующемся при значениях $n$, приближающихся к $10^{20} \mathrm{~cm}^{-3}, 1 / L=1 / h$, что в реальных условиях ниже первого значения на 2-3 порядка величины.

Как видно, полученные зависимости $1 / L$ от концентраций свободных носителей заряда в кристаллах кремния $p$ - и $n$-типа проводимости, отражающие предполагаемый ход эффективной температуры фронта реакции электрохимического порообразования, ведут себя разнонаправлено, так же как и размеры пор, образующихся в этих кристаллах. При этом значение $1 / L$ для слаболегированных кристаллов $n$-типа проводимости превышает соответствующие величины для кристаллов $p$-типа проводимости вплоть до $p \sim 2 \cdot 10^{19} \mathrm{~cm}^{-3}$, что также соответствует наблюдаемым концентрационным различиям темпов увеличения размеров пор с повышением плотности тока в кремнии $p$ - и $n$-типа проводимости (рис. 1).

Понятно, что вытекающие из предложенной модели процессов электрохимического порообразования в кремнии корреляции между эффективной температурой на фронте продвижения пор и их поперечными размерами могут пока рассматриваться исключительно с качественной стороны. Тем не менее выявленное в настоящей работе соответствие оцениваемой интенсивности тепловыделения в приповерхностных областях кристаллов $p$ и $n$-типа проводимости с различными уровнями легирования размерам пор, формирующихся в стационарных режимах анодного травления, может рассматриваться как дополнительное свидетельство справедливости развиваемых нами представлений о процессах анодного порообразования в полупроводниках, как о протекающих в импульсном режиме самоорганизующихся кооператив- ных реакциях, инициируемых падением потенциала в слое Гельмгольца, и непосредственно не обусловленных транспортом дырок из объема кристалла к реакционной поверхности.

\section{Заключение}

В работе приведены расчеты протяженностей областей рассеяния в кристаллах кремния энергии электронов, импульсно высвобождаемых на фронте кооперативной электрохимической реакции порообразования, протекающей на поверхности анодно- поляризованного полупроводника. Рассмотрены механизмы диссипации энергии неравновесных электронов в кристаллах $n$ и $p$-типа проводимости и показано, что зависимости глубины области выделения энергии инжектированных электронов от концентрации свободных носителей заряда в кристаллах кремния различного типа проводимости имеют противоположный характер. Выявлена однозначная корреляция между оцениваемой плотностью мощности тепловой энергии, выделяемой в прифронтальной области травящегося кристалла, и экспериментально найденными величинами поперечных размеров каналов, прорастающих в него пор. Таким образом, принятая нами модель анодного порообразования в алмазоподобных полупроводниках, как совокупности дискретных импульсных процессов кооперативного нуклеофильного замещения приповерхностных атомов решетки хемосорбированными анионами, позволила впервые предложить непротиворечивое качественное объяснение наблюдаемым зависимостям размеров пор в кристаллах кремния от типа и концентрации в них свободных носителей заряда, а также от плотности анодного тока.

\section{Финансирование работы}

Работа выполнена при поддержке РФФИ, грант № 16-29-0108 офи_м.

\section{Конфликт интересов}

Авторы заявляют, что у них нет конфликта интересов.

\section{Список литературы}

[1] Uhlir A. // Bell Syst. Tech. J. 1956. Vol. 35. P. 333-338.

[2] Canham L.T. Properties of Porous Silicon. / Ed. by L.T. Visible. London: INSPEC, 1997. P. 249-255.

[3] Kovalev D., Gross E., Kunzner N. et. al. // Phys. Rev. Lett. 2002. Vol. 89. P. 137401.

[4] Kovalev D., Timoshenko V.Y., Kunzner N. et. al. // Phys. Rev. Lett. 2001. Vol. 87. P. 68301.

[5] Улин В.П., Улин Н.В., Солдатенков Ф.Ю., Семенов А.В., Бобыль А.В. // ФТП. 2014. Т. 48. Вып. 9. С. 1243-1248.

[6] Ксенобонтова О.И., Васин А.В., Егоров В.В., Бобыль А.В., Солдатенков Ф.Ю., Теруков Е.И., Улин В.П., Улин Н.В., Киселев О.И. // ЖТФ. 2014. Т. 84. Вып. 1. С. 67-78. 
[7] Астрова Е.В., Федулова Г.В., Смирнова И.А., Ременюк А.Д., Кулова Т.Л., Скундин А.М. // Письма в ЖТФ. 2011. Т. 37. Вып. 15. С. 87-94.

[8] Zhang X.G. Electrochemistry of Silicon and Its Oxide. New York, Boston, Dordrecht, London, Moscow: Kluwer Academic Publishers, 2004. 510 p.

[9] Bisi O., Ossicini S., Pavesi L. // Surf. Sci. Rep. 2000. Vol. 38. P. 5-126.

[10] Korotcenkov G. Porous Silicon from Formation to Application. Formation and Properties. Vol. 1. Taylor \& Francis Group, LLC, 2016. 423 p.

[11] Lehmann V., Ronnebeck S. // J. Electrochem. Soc. 1999. Vol. 146. P. 2968-2975.

[12] Lehmann V., Stengl R., Luigart A. // Mater. Sci. Eng. B. 2000. Vol. 69-70. P. 11-22.

[13] Carstensen J., Christophersen M., Foll H. // Phys. Status Solidi A. 2000. Vol. 182. P. 63-69.

[14] Улин В.П., Конников С.Г. // ФТП. 2007. Т. 41. Вып. 7. C. 867-877.

[15] Улин В.П., Улин Н.В., Солдатенков Ф.Ю. // ФТП. 2017. Т. 51. Вып. 4. С. 481-496.

[16] Databases, Archives and Catalogs. New Semiconductor Materials: Characteristics and Properties. URL: http://www.matprop.ru/

[17] Грег C., Синг К. Адсорбция, удельная поверхность, пористость. М.: Мир, 1984. 306 с.

[18] Ландау Л.Д., Лифииц Е.М. Квантовая механика. М.: Физматлит, 1974. 296 с.

[19] Гантмахер В.Ф., Левинсон И.Б. Рассеяние носителей тока в металлах и полупроводниках. М.: Наука, 1984. 352 с. 